\title{
INTRODUCTION TO THE PEDIATRIC HOSPITAL MEDICINE CORE COMPETENCIES
}

\section{Background}

Pediatric Hospital Medicine continues to evolve as an area of specialization, with the refinement of a distinct knowledgebase and skill set focused on the provision of high quality general pediatric care in the inpatient setting. It is the latest site-specific specialty to emerge from the field of general pediatrics, following a course similar to that charted by pediatric emergency medicine and pediatric critical care medicine in recent decades. The growth of the field has been spurred by a number of factors, including the converging needs for a dedicated emphasis on patient safety, quality improvement, throughput management, and teaching in the inpatient setting.

The number of practicing pediatric hospitalists is estimated to be approximately 2500 and rapidly increasing. To meet the educational needs of this growing cohort of pediatricians, local, regional, and national continuing medical education offerings occur on a regular basis. Furthermore, at least ten fellowships dedicated to advanced training in pediatric hospital medicine have been developed at academic institutions across North America. Despite this, there has been an absence of an accepted and peer-reviewed framework for professional and curriculum development.

The Pediatric Hospital Medicine Core Competencies represent the first comprehensive attempt to more formally define the standards for the knowledge, skills, attitudes, and focus on systems improvements that are expected of all pediatric hospitalists, regardless of practice setting or location. It is the culmination of more than five years of planning, research, and development by the Society of Hospital Medicine Pediatric Core Curriculum Task Force, leaders within the Academic Pediatric Association and the American Academy of Pediatrics, and the editorial board. The competencies include contributions from over 80 pediatric hospitalists, content experts, and internal and external reviewers representing university and community hospitals, teaching and non-teaching programs, and key societies and agencies involved in child health from all geographic regions of the United States and Canada. A companion article to
Pediatric Hospital Medicine Core Competencies in this Supplement provides additional details regarding the project methodology.

\section{Purpose}

The Pediatric Hospital Medicine Core Competencies provide a framework for professional and curriculum development for all pediatric hospitalists. The framework is intended for use by hospital medicine program directors, directors of medical student clerkships, residency programs, fellowships, and continuing medical education, as well as other educators involved in curriculum development. The competencies do not focus on specific content, but rather general learning objectives within the skills, knowledge, and attitudes related to each topic. Attaining competency in the areas defined in these chapters is expected to require post-residency training. This training is most likely to be obtained through a combination of work experience, local mentorship, and engagement in specific educational programs or fellowship. Pediatric hospitalists, directors, and educators can create specific instructional activities and methods chosen to reflect the characteristics of the intended learners and context of the practice environment.

\section{Organization Structure}

The Pediatric Hospital Medicine Core Competencies consist of 54 chapters, divided into four sections - Common Clinical Diagnoses and Conditions, Specialized Clinical Services, Core Skills, and Healthcare Systems: Supporting and Advancing Child Health. Within each section, individual chapters on focused topics provide competencies in three domains of educational outcomes: the Cognitive Domain (Knowledge), the Psychomotor Domain (Skills), and the Affective Domain (Attitudes). To reflect the emphasis of hospital medicine practice on improving healthcare systems, a fourth section entitled Systems Organization and Improvement is also included. An attempt has been made to make the objectives "timeless", allowing for creation of curriculum that can be nimble and reactive to new discoveries. Highly specific temporal changes in medicine are purposefully excluded, and instead the focus is on the drivers for these changes or advancements. Phrases 
and wording were selected to help guide the learning activities most likely to achieve each competency and to reflect the varied roles that pediatric hospitalists have in different practice settings. In this document, the terms "child" and "children" include infants, children, adolescents, and young adults up to the age of 21 , in accordance of policies of the American Academy of Pediatrics. However, it is also understood that care is rendered in pediatric settings for patients who may surpass this upper age limit based on diagnosis or special healthcare needs. Finally, although the entire document can be a resource for comprehensive program development, each chapter is intended to stand alone and thus support curriculum development specific to the needs of individual programs.

\section{Conclusion and Acknowledgement}

The Pediatric Hospital Medicine Core Competencies are intended to provide standards for the knowledge, skills, and attitudes expected of all pediatric hospitalists and to provide a framework for ongoing professional and curriculum development for learners at all levels. We welcome feedback and evaluation from pediatric hospitalists and from all with whom we partner to improve the care for hospitalized children.

We wish to acknowledge the dedication of authors and associate editors, and the thoughtful review by the members of hospital organizations, accrediting bodies, and agencies listed in this supplement. This inaugural edition of the Pediatric Hospital Medicine Core Competencies should serve as the foundation from which the field of Pediatric Hospital Medicine will continue to evolve. We look forward with anticipation to future revisions as we reflect on our goals and advance our field.

The Pediatric Hospital Medicine Core Competencies Editorial Board

Erin Stucky, MD

Mary C Ottolini, MD, MPH

Jennifer Maniscalco, MD, MPH 